\title{
Klystron bunching with sustained electron acceleration in agriculture
}

\author{
Sergey Morozov ${ }^{1}$, Konstantin Kuzmin ${ }^{2,}$, Igor Pavlov ${ }^{1}$, Vladimir Reut ${ }^{1}$, and Vladimir \\ Vershinin $^{2}$ \\ ${ }^{1}$ Moscow State University of Technology and Management named after K.G. Razumovsky \\ (Smolensk branch), 77, Lenin str., 215100, Vyazma, Russia \\ ${ }^{2}$ Moscow State University of Civil Engineering, 26, Yaroslavskoye Shosse, 129337, Moscow, Russia
}

\begin{abstract}
This article discusses klystron bunching with post-acceleration, when bunchers are placed in front of the accelerating tube, and the electronic seals previously formed during the acceleration are ungrouped. The processes of acceleration and bunching of relativistic electron beams can be combined when placing the cavity resonators of the klystron buncher in the gaps between the electrodes of the accelerating tube. In this case, the grouping process occurs in accelerating gaps.
\end{abstract}

\section{Introduction}

As is known, the production of high-current relativistic electron beams with energies above $1 \mathrm{MeV}$ involves the use of multi-gap accelerating systems (accelerator tubes) [1]. Moreover, because of the danger of high-voltage breakdown, the average electric field strength is $5-30 \mathrm{kV} / \mathrm{cm}$, which determines the relatively large length (several meters) of the accelerator tube. The electron stream is grouped gradually, starting with low electron energies, and simultaneously with the accelerator. Due to this, it is possible to obtain an intensive grouping mode along the length of the accelerating tube with suppression of highspeed modulation by space charge forces. This ensures the creation of monoenergetic electronic seals of small length at the output to the energy extraction system [2].

In contrast to the usual klystron group, where the repulsive forces of the space charge increase, in the klystron group with simultaneous beam acceleration they decrease in the forming seal, due to the acceleration process, which makes it difficult to ungroup the electronic seals. This leads to a continuous increase in the plasma wavelength and, accordingly, to an increase in the distance at which velocity modulation transforms into density. Therefore, for more efficient bunching, the distance between adjacent resonators in the accelerating tube is selected to be sequentially increasing in the direction from the electron gun to the collector.

\footnotetext{
*Corresponding author: kuzmin_kostya@mail.ru
} 


\section{Methods}

The processes of klystron bunching of relativistic electron beams during its continuous acceleration are calculated by analytical methods based on the equation of oscillations of electron flows, or by numerical methods formed on discrete models of electron flow, which is discussed below [3].

The equation of motion of the electron layer (disk) in the relativistic case in the presence of an external electric field with a voltage $E_{z c}$ has the following form:

$$
\frac{d^{2} Z}{d t^{2}}=-\frac{l}{m_{0}}\left(l-\frac{v^{2}}{c^{2}}\right)^{3 / 2} E_{z p}+\frac{l}{m_{0}}\left(l-\frac{u^{2}}{c^{2}}\right)^{3 / 2} E_{z p}
$$

where $E_{z p}$ is the space charge field acting on the disk.

Field $E_{z p}$ is determined by the excess space charge arising inside the electronic seal during bunching, and is found by the integration of fields from all disks. The transition to a moving coordinate system allows one to use a quasistatic approximation, therefore, the expressions obtained by formally replacing the radial angle of the pipe passage $\xi_{a}=\omega_{a} / \nu_{0}$ by a value $\xi_{a} / \gamma_{0} \frac{\xi}{\gamma_{0}}$ are valid for the field $E_{z p}$.

In the presence of an accelerating field, a coordinate system moving with the speed of the average (unmodulated) electron should be chosen as a moving coordinate system.

\section{Results and discussions}

Therefore, the radial bunching angle must be formally replaced by the value $\xi_{a} / \gamma^{*}$ where $\gamma^{*}\left(1-\beta^{* 2}\right)^{-1 / 2}, \beta^{*}=\frac{v r}{c} ; v^{*}$ is the velocity of the unmodulated electron, which changes due to acceleration.

Passing to the dimensionless coordinate $\theta^{*}=\frac{\omega_{z}}{v_{0}}$ and the bunching angle $\xi=\omega \mathrm{\imath}-\omega \mathrm{l}_{0}$, substituting the expression for the space charge field $E_{z p}$ in (1), we obtain the oscillation equation [4]

$$
\frac{d^{2} \theta}{d \xi^{2}}=\left[1-\beta_{0}^{2}\left(\frac{d \theta}{d \xi}\right)^{2}\right]^{3 / 2} a_{q}^{2}\left(\theta-\theta^{*}\right)\left[1-\beta_{0}^{2}\left(\frac{d \theta}{d \xi}\right)^{2}\right]^{3 / 2} \frac{e E z c-v_{0}}{m_{0} c^{2} \beta^{2} u}
$$

where $\theta^{*}=\frac{\omega_{z}}{v_{0}} ; z^{*}$ is the unmodulated layer coordinate; $\beta_{0}=\frac{v_{0}}{c} ; v_{0}$ is the velocity of an unmodulated electron before the start of an accelerating field.

To solve this equation of oscillations, it is necessary to know the dependence of the velocity change and the coordinates of the unmodulated layer.

The equation of motion of an unmodulated disk (when $E_{z p}=0$ ) has the following form: 


$$
m_{0} \frac{d}{d t}\left(\frac{v^{*}}{1-\left(v^{*} / c\right)^{2}}\right)=e E z c
$$

It is convenient to represent the first and second integrals of the equation in a dimensionless form

$$
\beta^{*}=\frac{\beta_{0} \gamma_{0}(1+H S)}{\sqrt{1+\beta_{0}^{2} v_{0}^{2}(1+H S)^{2}}} .
$$

In this case

$$
\begin{gathered}
H=\frac{\gamma_{0}}{\gamma_{0}+1} \frac{E_{z c} \nu_{0}}{v_{0} \omega} \\
\theta^{*}=\frac{c}{v_{0}} \int_{0}^{9} \beta^{*} d S=\frac{\omega \cup_{0}}{\left(\gamma_{0}-t\right) \cup E_{z c}} \sqrt{1+\beta_{0}^{2} \gamma_{0}^{2}(1+H S)^{2}} .
\end{gathered}
$$

The oscillation equation (2), taking into account the analytical dependencies (4) and (5), describes the process of grouping the relativistic electron beam during its acceleration. By solving this equation, it is possible to determine the trajectories of the extreme layers of the forming electronic seal and the magnitude of its greatest compression [5]. Approximating a real electronic seal within a single period by a rectangular current pulse, one can determine the amplitude of the convection current harmonic, as well as the magnitude of the averaged electron current in the seal and outside it. For single-stage bunching, when an electron beam unmodulated in density is grouped, the amplitudes of the harmonics of the convection current are determined by the initial and final lengths of the electron seal:

$$
\frac{I_{m n}}{I_{0}}=\frac{2}{n} \frac{\theta \cdot \sin \left[n \theta \frac{\theta_{0}-\theta}{\theta_{0}}\right]}{\left(\theta_{0}-\theta\right)[\pi-(\theta-\theta)]} .
$$

In cascade bunching, it is necessary to take into account the change in the initial seal length due to the involvement of additional electrons in the forming bunch at the modulation of the electron beam in intermediate resonators [6].

The klystron bunching process with continuous electron acceleration can also be investigated by numerical methods based on the discrete model of the relativistic electron beam. In the presence of an accelerating field, the equations of motion have the following form [8]:

$$
\begin{gathered}
\frac{d^{2} \theta_{1}}{d \zeta^{2}}+\frac{\pi}{N} a_{p}^{2}\left[1-\beta_{0}^{2}\left(\frac{d \theta_{i}}{d \zeta}\right)^{2}\right]_{\neq j}^{3 / 2} \sum_{=i}\left[F_{i j} \operatorname{sign}\left(\theta_{i}-\theta_{j}\right)\right]-\left[1-\beta_{0}^{2}\left(\frac{d \theta_{i}}{d \zeta}\right)\right]^{3 / 2} \times \\
\frac{e E z c v_{0}}{m_{0} c^{2} \beta_{0}^{2} \omega}=0,
\end{gathered}
$$


where $\beta_{0}=\frac{\mathrm{v}_{0}}{C} ; \mathrm{v}_{0}$ is the velocity of an unmodulated electron before the start of an accelerating field.

Figure 1, a shows dependences of the relative amplitude of the first harmonic of the convection current $I_{m i} / I_{0}$ of bunching angle $\zeta$ at $a_{p}=0,39, \frac{M v_{m}}{v_{0}}=0,2$ and various values of the electric field strength $E_{z c}=0 ; 650 \mathrm{kV} / \mathrm{m} ; 1300 \mathrm{kV} / \mathrm{m}$ (curves 1,2 , and 3, respectively).

The presence of an accelerating field prevents the ungrouping of bunches, since the forces of the space charge in the compaction decrease with beam energy increasing. In addition, at high accelerating voltages, the bunching angle increases, at which the maximum of the first harmonic of the convection current ( $\left.I_{m i} / I_{0}\right)_{\max }$ is reached.

During bunching in an accelerating field, a sufficiently dense clot forms, which does not "fall apart" at considerable drift lengths and, accordingly, the magnitude of the first harmonic is almost unchanged. This is confirmed by the phase trajectories of grouping electrons for $E_{z c}=650 \mathrm{kV} / \mathrm{m}$ (Figure 1, b).
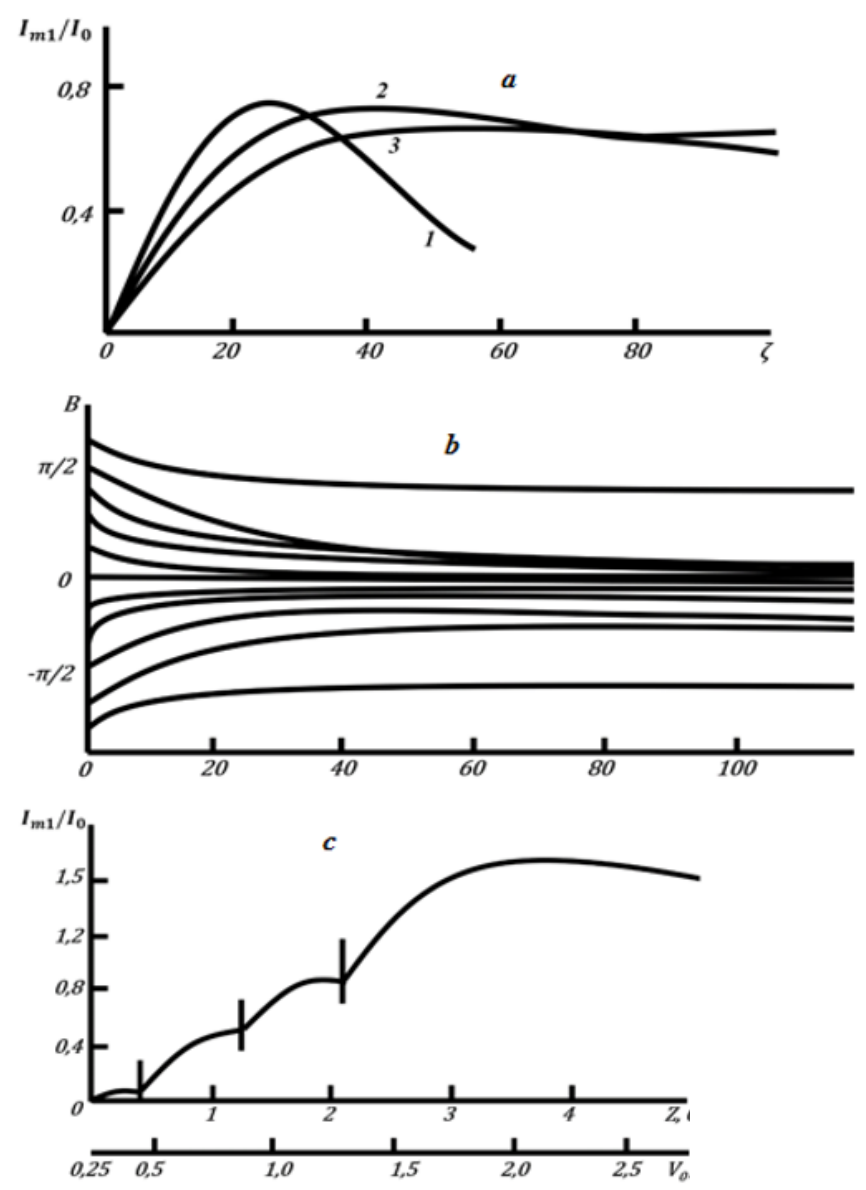

Fig. 1. Phase trajectories and a change in the coefficient of the first harmonic of the convection current for an electron beam in the bunching mode with continuous acceleration. 
The considered method of klystron bunching with continuous acceleration is studied in relation to the accelerator. This accelerator is designed for an electron energy of 2-8 MeV. The electron gun forms a beam with $I_{0}=150-200 A, I_{\text {om }}=250-300 \mathrm{kV}$, which accelerates in a high-voltage tube containing 12-14 accelerating modules $30 \mathrm{~cm}$ long. The beam energy at the output of the accelerator is determined by the magnitude of the accelerating voltages on the modules. The continuous-acceleration klystron buncher of relativistic electron beam is a set of resonators embedded between accelerating modules [9].

As an example, we give data on a $2 \mathrm{MeV}$ energy buncher, consisting of an input and three intermediate resonators. The parameters of the electron beam are as follows: $I_{0}=150 \mathrm{~A}, U_{m i}=250 \mathrm{kV}$, the radii of the beam and the drift tube $b=0.75 \mathrm{~cm}, a=1.1$

$\mathrm{cm}$, respectively, the intensity of the accelerating field $E_{z c}=500 \mathrm{kV} / \mathrm{m}$, the beam power at the output of the accelerator $P_{o}=0.3 \mathrm{GW}$.

The amplitudes of the modulating voltages on the resonators are $1 ; 47 ; 117$; and 333 $\mathrm{kV}$, and the lengths of the cascades are 40, 83, 85, and $104 \mathrm{~cm}$, respectively. Such a buncher device forms electron bunches with a relative amplitude of the first harmonic of the convection current $I_{m i} / I_{0}=1.8$. A change in the magnitude $I_{m i} / I_{0}$ along the axis of a buncher is shown in Figure 1, c. The dotted line indicates the position of the intermediate resonators. The small voltage amplitude at the output resonator $U_{m i}=4 \mathrm{kV}$ makes it possible to limit the input power to $\sim 1 \mathrm{~kW}$. The values of the phase angles of detuning $\varphi_{i}$ and frequency detuning $\Delta \omega i / \omega_{0}$ of the intermediate resonators are given in Table 1 .

Table 1. The values of the phase angles of detuning and frequency detuning of the intermediate resonators.

\begin{tabular}{|c|c|c|c|c|c|}
\hline$U_{m i}, \mathrm{kV}$ & $\varphi_{i}$ & $\Delta \omega i / \omega_{0}$ & $M_{i}$ & $Q_{n i}$ & $\frac{I_{m i}}{I_{0}}$ \\
\hline 47 & 70 & $3.98^{*} 10^{-3}$ & 0.95 & 343 & 0.08 \\
\hline 117 & 82.5 & $1.04^{*} 10^{-2}$ & 0.98 & 371 & 0.48 \\
\hline 333 & 86.5 & $2.15^{*} 10^{-2}$ & 0.99 & 386 & 0.92 \\
\hline
\end{tabular}

It should be noted that because of the danger of breakdown, it is necessary to use three resonators with an amplitude at each $111 \mathrm{kV}$ instead of the third intermediate resonator. The values of the impact coefficients $M_{i}$ and loaded $\mathrm{Q}$ factors of the resonators $Q_{n i}$ are calculated taking into account changes in the start-up energy along the axis of the buncher.

With a further increase in the acceleration rate to $\sim 1500 \mathrm{kV} / \mathrm{m}$, the electron energy can be increased to $\sim 6 \mathrm{MeV}$, and the output power will reach a gigawatt level. In order to maintain the efficiency of the klystron bunching of the relativistic electron beam, the system of modulating resonators should be shifted closer to the start of the accelerator tube.

\section{Conclusions}

The development of new areas of science and technology, such as radiolocation of space objects, long-range space communications systems, energy transmission over long distances via a radio beam, thermonuclear fusion, accelerator technology and others requires a significant increase in output power and efficiency of microwave devices. One of the ways to solve this problem is the use of high-current relativistic electron beams. 
At present, in Russia and abroad, there are being conducted intensive theoretical and experimental studies on the use of high-current relativistic electron beams for the generation and amplification of microwave oscillations in order to achieve a terawatt power level. These studies are aimed both at developing fundamentally new methods for generating microwave radiation and at creating relativistic analogues of traditional microwave devices.

\section{References}

1. K.A. Kuzmin, S.M. Morozov, A.V. Rogozhina, Agrarian Scientific Journal 3, 74-79 (2019)

2. K.A. Kuzmin, S.M. Morozov, Engineering Herald of the Don 3, 75-83 (2018)

3. S. Morozov, K. Kuzmin, G. Makarov, MATEC Web Conference 193, 102-115 (2018)

4. S.M. Morozov, V.V. Efimov, K.A. Kuzmin, E.V. Balmashnova, L.I. Kochetkova, Bulletin of construction equipment 9, 37-49 (2018)

5. L. Landau, E. Lifchic, The theory of a field (Moscow, 1988)

6. H. Kroemer, Electrical and Computer Engineering Department (University of California, Santa Barbara, 2001)

7. V.I. Fedulov, ARW977788 Emerging Applications of Vacuum-Arc-Produced Plasma (Ion and Electron Beams, Dordrecht, 2003)

8. V.I. Fedulov, S.M. Morozov, Modern television and radio electronics, 299-301 (2011)

9. V.I. Fedulov, S.M. Morozov, Modern television and radio electronics, 301-304 (2011) 